\title{
Hyperplastic Polyp with Epithelial Misplacement (Inverted Hyperplastic Polyp): A Clinicopathologic and Immunohistochemical Study of 19 Cases
}

\author{
Rhonda K. Yantiss, M.D., Harvey Goldman, M.D., Robert D. Odze, M.D., F.R.C.P.C. \\ Department of Pathology, Beth Israel Deaconess Medical Center (RKY, HG) and Department of Pathology, \\ the Brigham and Women's Hospital, Harvard Medical School (RDO), Boston, Massachusetts
}

Hyperplastic polyps of the colon are the most common type of benign colonic polyp. Rarely, these polyps may show misplaced epithelium within the submucosa, thereby simulating an adenoma with pseudoinvasion or even an adenocarcinoma. In this study, we describe the clinical, pathologic, and immunophenotypic features of 19 hyperplastic polyps with misplaced epithelium to identify potential diagnostic pitfalls and gain insight into their pathogenesis. Routinely processed polypectomy specimens from 12 patients with 19 hyperplastic polyps containing foci of misplaced epithelium were evaluated for a variety of morphologic features including pattern and extent of submucosal involvement, continuity of the submucosal epithelium with the mucosa, presence of recent or remote hemorrhage, inflammation, association of misplaced epithelium with lymphoid aggregates, inflammation, and defects in the muscularis mucosae. Clinical and endoscopic data were obtained and correlated with the histologic findings. Immunoperoxidase stains (ABC method) for collagen IV (basement membrane marker), MIB-1 (proliferation marker), and E-cadherin (intercellular adhesion protein) were performed in all cases. The study group consisted of five males and seven females ranging in age from to 52 to 73 years (mean: $63 \mathrm{y}$ ). All of the polyps were located in the rectum or sigmoid colon, and their mean size was $0.5 \mathrm{~cm}$ (range: 0.2 to $1.0 \mathrm{~cm}$ ). Most showed misplaced epithelium in a lobular $(26 \%)$ or a mixed pattern consisting of lobules and irregularly distributed crypts (63\%) that, upon deeper levels, was almost always continuous with the mucosal portion of the polyps (95\%). Defects in the muscularis mucosae and splaying of the muscle fibers

Copyright (C) 2001 by The United States and Canadian Academy of Pathology, Inc.

VOL. 14, NO. 9, P. 869, 2001 Printed in the U.S.A.

Date of acceptance: April 18, 2001

Address reprint requests to: Robert D. Odze, M.D., Department of Pathol ogy, Brigham and Women's Hospital, 75 Francis Street, Boston, MA 02215; e-mail: rdodze@bics.bwh.harvard.edu; fax: (617) 277-9015. around misplaced epithelium were seen in all cases. Lymphoid aggregates were present adjacent to foci of misplaced epithelium in $37 \%$ of cases. Fresh hemorrhage, vascular congestion, and hemosiderin deposits were present in 79,53 , and $42 \%$ of cases, respectively. Strong and uniform staining of the misplaced epithelium for MIB-1 and E-cadherin was demonstrated in all cases, similar to that seen in the lower third of the mucosal portion of the polyps. A continuous collagen IV basement membrane pattern of staining was noted around all foci of misplaced epithelium. Hyperplastic polyps with misplaced epithelium probably occur secondary to trauma-induced protrusion of glands through breaks in the muscularis mucosae. Pathologists should be aware of this entity to avoid diagnostic confusion with other, more serious lesions, such as adenomas with pseudoinvasion or well-differentiated adenocarcinoma.

KEY WORDS: Hyperplastic polyp, Immunohistochemistry, Inverted, Misplaced epithelium, Pseudoinvasion.

Mod Pathol 2001;14(9):869-876

Hyperplastic polyps are the most common type of colonic polyp. The prevalence of these lesions ranges from 25 to $80 \%$ in the general population (1-6). Hyperplastic polyps are typically small $(<5$ $\mathrm{mm})$, smooth, sessile lesions located predominantly in the left colon of patients usually $>40$ years of age $(7,8)$. Morphologically, hyperplastic polyps are characterized by numerous crypts with a convoluted lumenal pattern of growth and are typically lined by absorptive cells with a variable number of goblet cells and endocrine cells $(9,10)$. Although hyperplastic polyps may occasionally grow to large sizes and the crypts may harbor frequent mitoses, the cytologic features of the cells are bland and thus remain distinct from adenomatous lesions with a serrated contour (serrated adenoma). 
We have noted that hyperplastic polyps may show crypts located within the muscularis mucosae or submucosa of the polyp. When pronounced, these features may cause diagnostic confusion with other lesions such as prolapse-type inflammatory polyps, adenomas with epithelial misplacement, or even well-differentiated adenocarcinoma. These lesions, termed "inverted hyperplastic polyps" by others $(11,12)$, have received little attention in the literature, and as a result, their pathogenesis is poorly understood.

Therefore, the purpose of this study was to evaluate the clinical and morphologic features of 19 hyperplastic polyps with misplaced epithelium. Cases were also immunohistochemically stained with antibodies to collagen IV (basement membrane), E-cadherin (intercellular adhesion protein), and MIB-1 (proliferation marker) to demonstrate the noninvasive, yet proliferative, nature of the misplaced epithelium and to gain insight into the pathogenesis of epithelial misplacement in these lesions.

\section{MATERIALS AND METHODS}

\section{Study Group}

The study group consisted of 12 patients, all of whom had at least one hyperplastic polyp that showed misplaced epithelium within the submucosa of the polyp. All cases consisted of polypectomy samples obtained as a result of routine colonoscopy or sigmoidoscopy performed for a variety of presenting complaints. All of the cases, except one, were retrieved from the surgical pathology files of the Brigham and Women's Hospital and the Beth Israel Deaconess Medical Center (Boston, MA) over a 4-year period from 1997 to 2000. One case was retrieved from the personal consult files of one of the authors (RDO), in which the referring pathologist entertained a diagnosis of adenocarcinoma in a serrated adenoma. For all patients, pertinent clinical information was obtained by a review of the patients' endoscopy reports and medical records. The following data were obtained: patient age, gender, medical history, and endoscopic findings, including polyp size and location.

\section{Histologic Evaluation}

Nineteen hyperplastic polyp specimens from 12 patients were routinely processed, fixed in $10 \%$ buffered formalin, embedded in paraffin, and stained with hematoxylin and eosin (H\&E). The following morphologic features were evaluated: the architectural growth pattern and extent of submucosal involvement by misplaced crypts, the presence or absence of continuity of the submucosal crypts with the mucosal epithelium, the presence of recent (fresh blood) or remote (hemosiderin) hemorrhage, vascular congestion, cytologic features of the epithelial cells in the misplaced crypts, the presence or absence of lymphoid nodules and mucin extravasation in association with the misplaced crypts, inflammation, and the presence or absence of discontinuity of the muscularis mucosae adjacent to misplaced epithelium.

\section{Immunohistochemical Evaluation}

Immunoperoxidase studies were performed on $4-\mu \mathrm{m}$-thick formalin-fixed, paraffin-embedded tissue sections using the standard avidin-biotin complex (ABC) technique. The slides were incubated for 1 hour at $60^{\circ} \mathrm{C}$, rehydrated with xylene (four washes, $3 \mathrm{~min}$ each), 100\% ethanol (four washes, 3 min each), and distilled water (5 min), and pretreated for endogenous peroxidase activity (3\% hydrogen peroxide/methanol) for 30 minutes, followed by a rinse in running water for 5 minutes. Slides to be stained with E-cadherin and MIB-1 (Ki-67) were microwave pretreated $(800 \mathrm{~W})$ at $93^{\circ} \mathrm{C}$ for 30 minutes in preheated $10 \mathrm{~mm}$ citrate buffer ( $\mathrm{pH}$ 6.0). The slides were then cooled for 15 minutes at room temperature and transferred to phosphate buffered saline (PBS). Slides to be stained with collagen IV were digested with protease XXIV $(0.01 \%$ prewarmed $0.005 \mathrm{~m}$ Tris buffered saline, $\mathrm{pH}$ 7.6) at $37^{\circ} \mathrm{C}$ for 10 minutes, rinsed in running water, and transferred to PBS. All tissue sections were then treated with $1.5 \%$ horse serum (15 $\mathrm{min}$ at room temperature). The slides were incubated in a humid chamber at room temperature for 1 hour with the primary antibodies: E-cadherin (HECD-1, 1:2000 dilution, Zymed Laboratories, Inc., San Francisco, CA), Ki-67 (monoclonal MIB-1, 1:200 dilution, Immunotech, Division of Coulter Corporation, Westbrook, ME), and collagen IV (CIV 22, 1:50 dilution, DAKO, Carpinteria, CA). The secondary antibody (Vectastain Elite ABC Kit, Vector Laboratories, Inc., Burlington, CA) was used according to the manufacturer's instructions. The slides were rinsed with PBS between incubations. Finally, the tissue sections were developed using 3,3'-diaminobenzidine (3,3'-diaminobenzidine) as the substrate (Sigma Chemical Co., St. Louis, MO) and counterstained with Gill's hematoxylin (Fisher Scientific, Pittsburgh, PA). Positive controls included human skin for collagen IV, human tonsillar tissue for MIB-1, and invasive ductal carcinoma of the human breast for E-cadherin. Negative controls for each antibody consisted of incubating the slides with $1.5 \%$ horse serum in the absence of the primary antibody.

The degree of collagen IV staining of the basement membrane surrounding the crypts and blood vessels was recorded as continuous, discontinuous, 
or absent. The degree of MIB-1 staining of the crypts was evaluated by noting the location and extent of nuclear staining in the mucosal and submucosal epithelium. Cytoplasmic and/or membranous E-cadherin staining were assessed as strong, weak, or absent in comparison to controls. For all stains, the mucosal and submucosal epithelium were evaluated separately. The histologic and immunohistochemical features were evaluated by two of the authors (RKY and RDO) without any notable discrepancies in interpretation.

\section{RESULTS}

\section{Clinical and Endoscopic Features:}

A summary of the clinical and endoscopic data is listed in Table 1. The study group consisted of five males and seven females ranging in age from to 52 to 73 years (mean age: $63 \mathrm{y}$ ). The clinical indication for colonoscopy in each patient was a history of polyps (four patients), a family history of carcinoma (two patients), routine screening (two patients), gastrointestinal bleeding (one patient), occult blood positivity (one patient), abdominal pain (one patient), or the presence of a concurrent adenocarcinoma (one patient). Eleven patients (92\%) underwent full colonoscopy, and one (8\%) underwent flexible sigmoidoscopy.

Three patients, one of whom was evaluated by sigmoidoscopy, had a single hyperplastic polyp with misplaced epithelium and no other lesions noted upon examination. Of the remaining nine patients, two had a solitary hyperplastic polyp with epithelial misplacement in addition to either an adenoma (one patient) or an invasive adenocarcinoma (one patient). Seven had multiple hyperplastic polyps (mean: 3 , range: 2 to 4 ): three had multiple hyperplastic polyps, each containing areas of

TABLE 1. Clinical and Endoscopic Features of the 12 Study Patients

\begin{tabular}{lc}
\hline \multicolumn{1}{c}{ Feature } & Result \\
\hline Male/female ratio & $5 / 7$ \\
Mean age (y) & 63 \\
Indication for colonoscopy & 4 \\
Polyps & 2 \\
Family history of colon cancer & 2 \\
Screening & 1 \\
Rectal bleeding & 1 \\
Occult blood & 1 \\
Colonic adenocarcinoma & 1 \\
Abdominal pain & \\
Location of polyp & 11 \\
Sigmoid & 8 \\
Rectum & 0.5 \\
Mean size (cm) & \\
Associated lesions & 8 \\
Hyperplastic polyp & 5 \\
Adenoma & 2 \\
Colonic adenocarcinoma & \\
\hline
\end{tabular}

misplaced epithelium (two had two polyps and one had three polyps), two had a single hyperplastic polyp with misplaced epithelium in addition to conventional hyperplastic polyps and a single adenoma, one had four hyperplastic polyps with misplaced epithelium and an adenoma, and one had a single hyperplastic polyp with misplaced epithelium in addition to three conventional hyperplastic polyps, an adenoma, and an invasive adenocarcinoma elsewhere in the colon. All 19 hyperplastic polyps with misplaced epithelium $(100 \%)$ were located in the rectum (8 patients) or sigmoid colon (11 patients) and were sessile in appearance. None were pedunculated. The mean size of the hyperplastic polyps with misplaced epithelium was 0.5 cm (range: 0.2 to $1.0 \mathrm{~cm}$ ).

\section{Pathologic Features}

The pathologic features of the study cases are summarized in Table 2. Overall, three architectural growth patterns of misplaced epithelium were noted in the muscularis mucosae or submucosa of the hyperplastic polyps. One pattern showed welldelineated lobules of crypts $(n=5)$, each surrounded by abundant lamina propria. A second growth pattern demonstrated poorly circumscribed aggregates, or individual irregularly distributed crypts $(n=2)$. The remaining cases $(n=12)$ demonstrated a combination of these two patterns (Fig. 1). In all cases, the misplaced crypts were surrounded by a discrete rim of lamina propria. Morphologically, the crypt epithelium was comprised predominantly of mucin-depleted goblet cells containing basally located nuclei and scattered typical mitotic figures indistinguishable from that seen in the lower third of the intramucosal component of the hyperplastic polyps (Fig. 2). However, in some cases (32\%), the centrally located submucosal crypts contained goblet cells with abundant mucin and small nuclei similar to those seen on the mucosal surface of the polyp. Mild cytologic atypia, characterized by slightly enlarged oval to round nuclei, nuclear pseudostratification, and promi-

TABLE 2. Pathologic Features of Hyperplastic Polyps with Misplaced Epithelium

\begin{tabular}{lc}
\hline \multicolumn{1}{c}{ Feature } & Number of Cases (\%) \\
\hline Architectural growth pattern & \\
$\quad$ Lobular & $7(26)$ \\
Irregular & $2(11)$ \\
Mixed & $12(63)$ \\
Defect in muscularis mucosae & $19(100)$ \\
Fibromuscular splaying & $19(100)$ \\
Lamina propria surrounding misplaced epithelium & $19(100)$ \\
Continuity with mucosal epithelium & $18(95)$ \\
Vascular congestion & $10(53)$ \\
Recent hemorrhage & $15(79)$ \\
Hemosiderin & $8(42)$ \\
Lymphoid aggregates & $7(37)$ \\
\hline
\end{tabular}




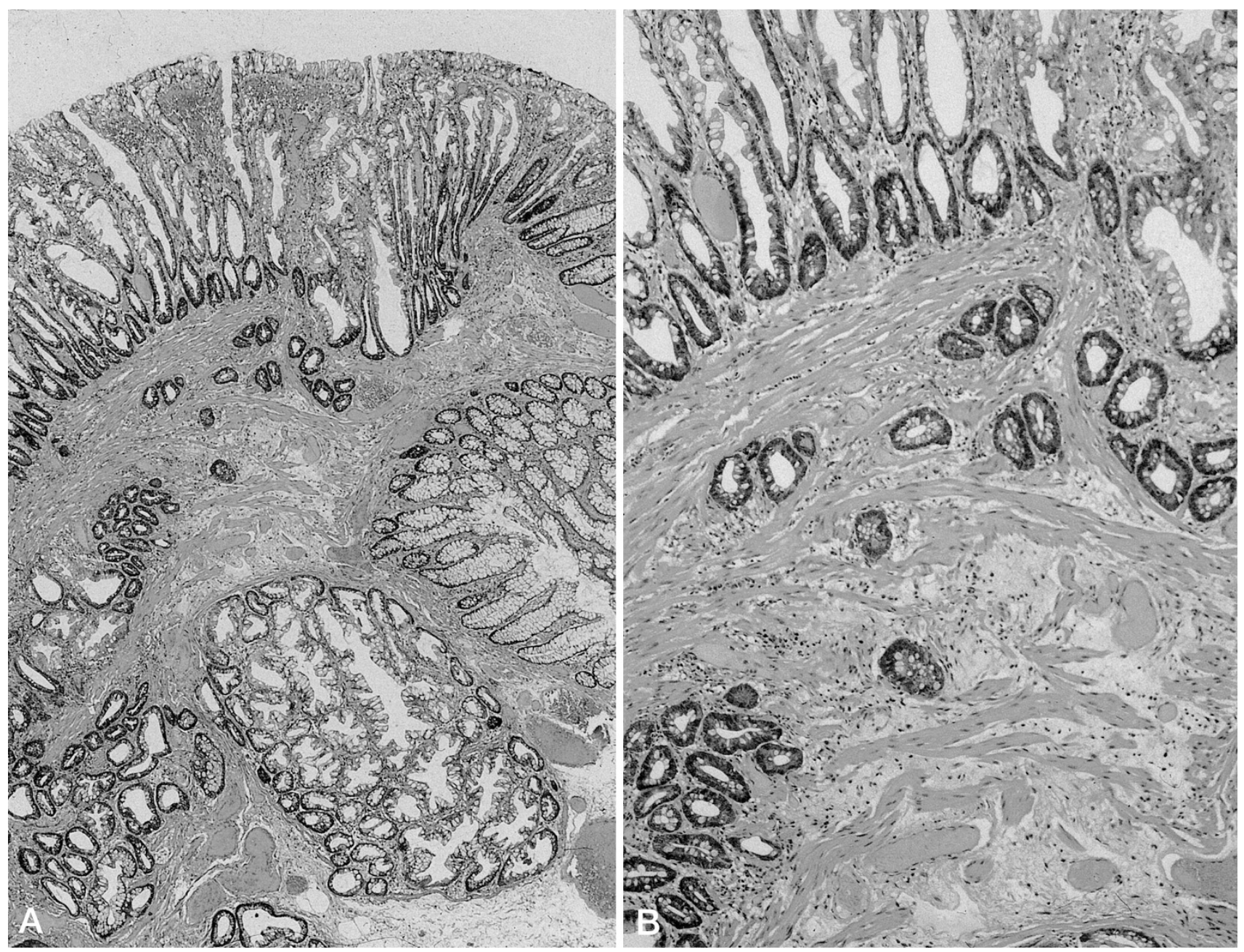

FIGURE 1. A, low-power view of a hyperplastic polyp with misplaced epithelium. The submucosa of the polyp shows a mixture of well-demarcated lobules and irregular aggregates of hyperplastic crypt epithelium. Misplaced epithelium is present both within and beneath strands of muscularis mucosae. B, high-power view of the superficial submucosa showing strands of muscularis mucosae surrounding crypt epithelium.

nent, occasionally multiple, nucleoli, was present in all cases (Fig. 2). However, frankly dysplastic cytologic features such as clumped chromatin or nuclear membrane irregularities were not observed in any of the cases.

Overlying areas of epithelial misplacement, the muscularis mucosae was discontinuous in all cases. In 7 of 19 cases (37\%), the areas of discontinuous muscularis mucosae occurred adjacent to lymphoid aggregates (Fig. 3), whereas in the remainder, these aggregates were not detected even upon deeper sectioning of the tissue blocks. As a result, splaying of smooth muscle fibers was often noted surrounding lobules of misplaced crypts (Fig. 4). Continuity of the misplaced epithelium with the epithelium in the mucosa was demonstrated upon deeper sectioning of the tissue blocks in 18/19 (95\%) cases. Vascular congestion $(53 \%)$, fresh hemorrhage (79\%), and hemosiderin deposits (42\%; as confirmed with a Pearl's prussian blue iron stain), suggestive of tissue injury, were present in the polyps of many cases. In fact, hemosiderin deposits were always associated with areas of fresh hemorrhage (Fig. 3). However, these features were absent from the adjacent nonpolypoid mucosa, when present. A mild degree of mononuclear cell inflammation was present in the lamina propria surrounding the misplaced crypts in all cases but was comparable in quantity and quality to that seen in the lamina propria in the mucosa. Features commonly seen in colonic prolapse-type polyps, such as cryptitis, neutrophilic inflammation, fibromuscular hyperplasia of the lamina propria, and surface erosions, degenerative, or regenerative changes, were absent in all cases. Stromal desmoplasia and mucin extravasation were absent in all cases.

\section{Immunohistochemical Features}

Diffuse cytoplasmic and membrane staining for E-cadherin was seen in the intramucosal and submucosal component of all hyperplastic polyps (100\%). For cases in which normal colonic mucosa was also present, the distribution and intensity of epithelial staining was similar to that observed in the polyps. 


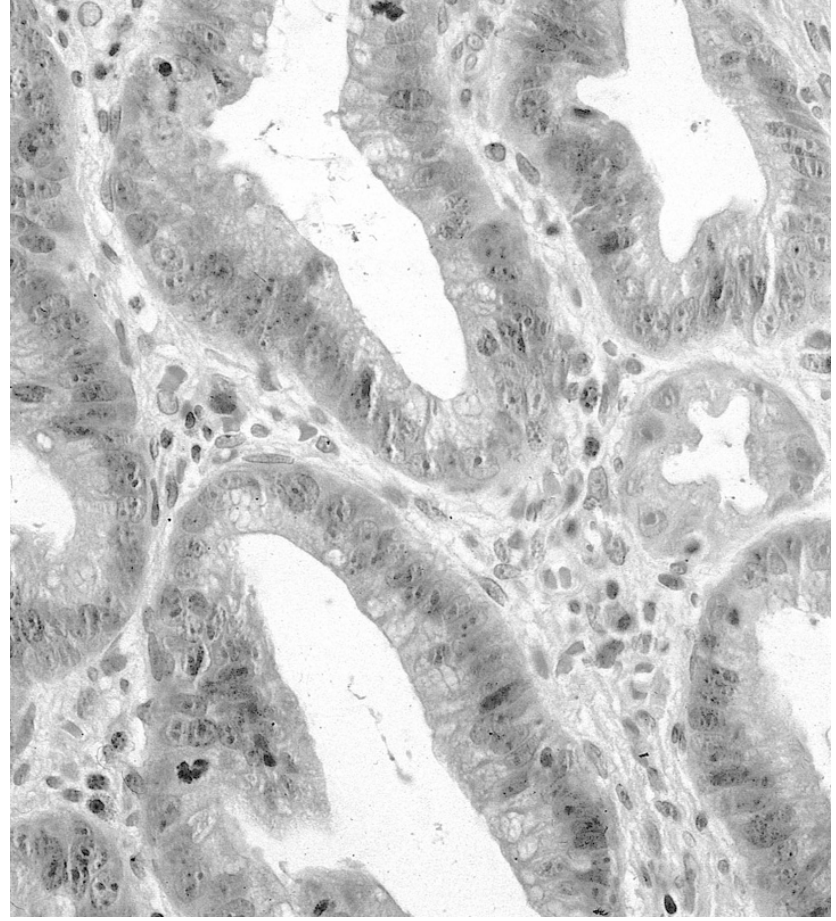

FIGURE 2. High-power view of misplaced crypt epithelium in the submucosa of a hyperplastic polyp. The crypts are surrounded by a small amount of lamina propria containing lymphocytes, plasma cells, and eosinophils. The epithelium is composed of mucin-depleted cells with slightly enlarged round to oval nuclei containing one or more prominent nucleoli and an open chromatin pattern. Several nonatypical mitotic figures are present.

The degree and location of MIB-1 staining in areas of misplaced epithelium were similar to those observed in the mucosal component of the polyps. The submucosal crypt epithelium showed strong, diffuse nuclear staining (mean: 81 positive nuclei per 100 cells) similar in intensity and quantity to that observed in the lower third of the intramucosal crypts (mean: 81 positive nuclei per 100 cells; Fig. 5). In contrast, the centrally located submucosal crypt epithelium, an area that morphologically resembled the surface epithelium of the polyps, was largely negative for MIB-1, similar to hyperplastic epithelium at the surface of the polyps (mean: 7 positive nuclei per 100 cells). Normal colonic mucosa, when present, demonstrated minimal MIB-1 staining of the surface epithelium and numerous positive cells in the lower third of the mucosa.

A continuous, noninterrupted basement membrane pattern of collagen IV staining was present surrounding all foci of misplaced epithelium, similar to crypts in the mucosa of the hyperplastic polyps (Fig. 6). A similar pattern of staining was present surrounding blood vessels in the submucosa and mucosa as well. Basement membrane staining was also demonstrated surrounding crypt epithelium and blood vessels in the adjacent normal colonic mucosa, where present.

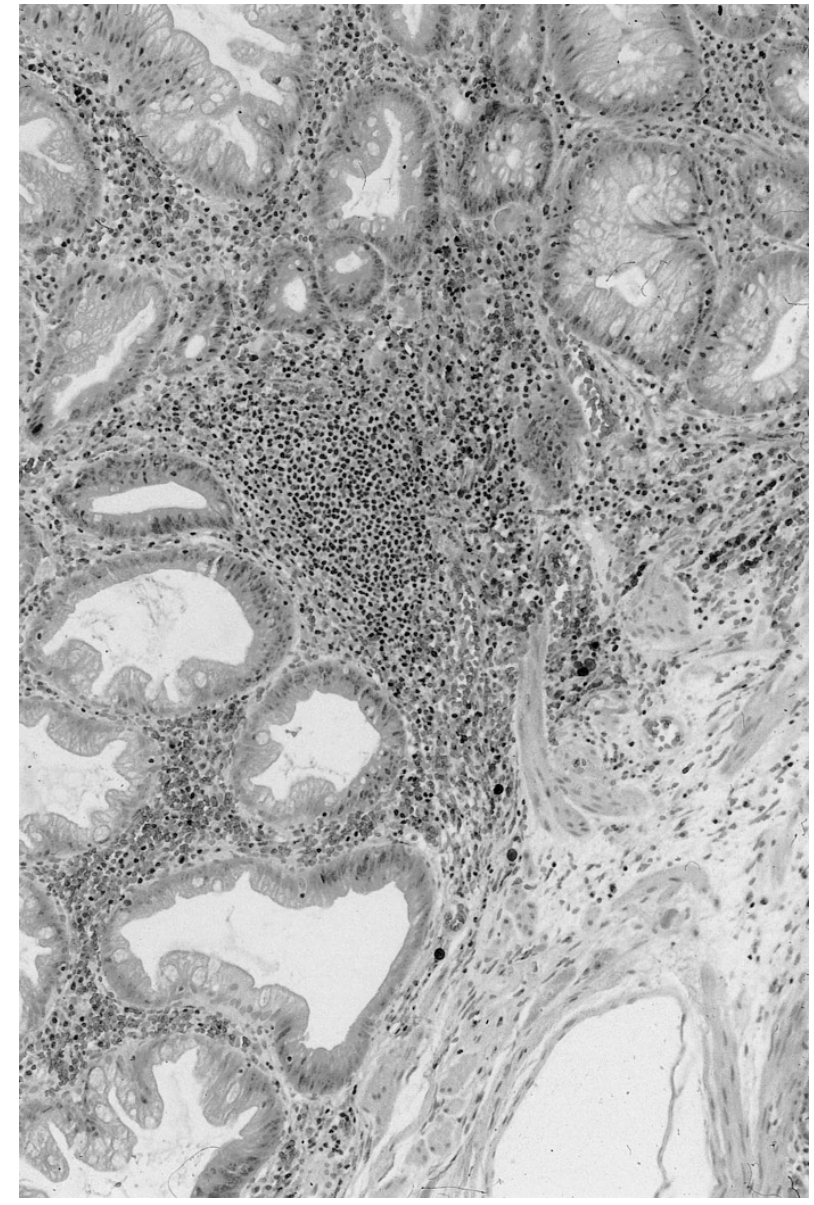

FIGURE 3. High-power view of a hyperplastic polyp with epithelial misplacement. Hemosiderin deposits, fresh hemorrhage, and a lymphoid aggregate are associated with an area of misplaced epithelium (left) at the junction of the mucosa and submucosa. Notice that the muscularis mucosae (right) is interrupted at this location.

\section{DISCUSSION}

In this report, we described the demographic, pathologic, and selected immunophenotypic features of 19 hyperplastic polyps that contained misplaced epithelium in the submucosa. Clinically, the lesions developed in males and females nearly equally and were located exclusively in the left colon. Conventional hyperplastic polyps are reported to occur more frequently in males but are known to arise more commonly in the left colon $(7,8)$. Pathologically, the polyps were characterized by submucosal misplaced epithelium arranged in well-delineated lobules, and/or irregular congeries of crypts, within the submucosa that were morphologically similar to that seen in the lower third of the mucosal portion of the lesions, and often associated with breaks in the muscularis mucosae. Misplaced epithelium was surrounded by a continuous basement membrane, as demonstrated by collagen IV staining, and had a high MIB-1 proliferation index, similar to that observed in the mucosal crypt bases. E-cadherin staining was similar to that seen in the normal colonic mucosa. Thus, the mor- 


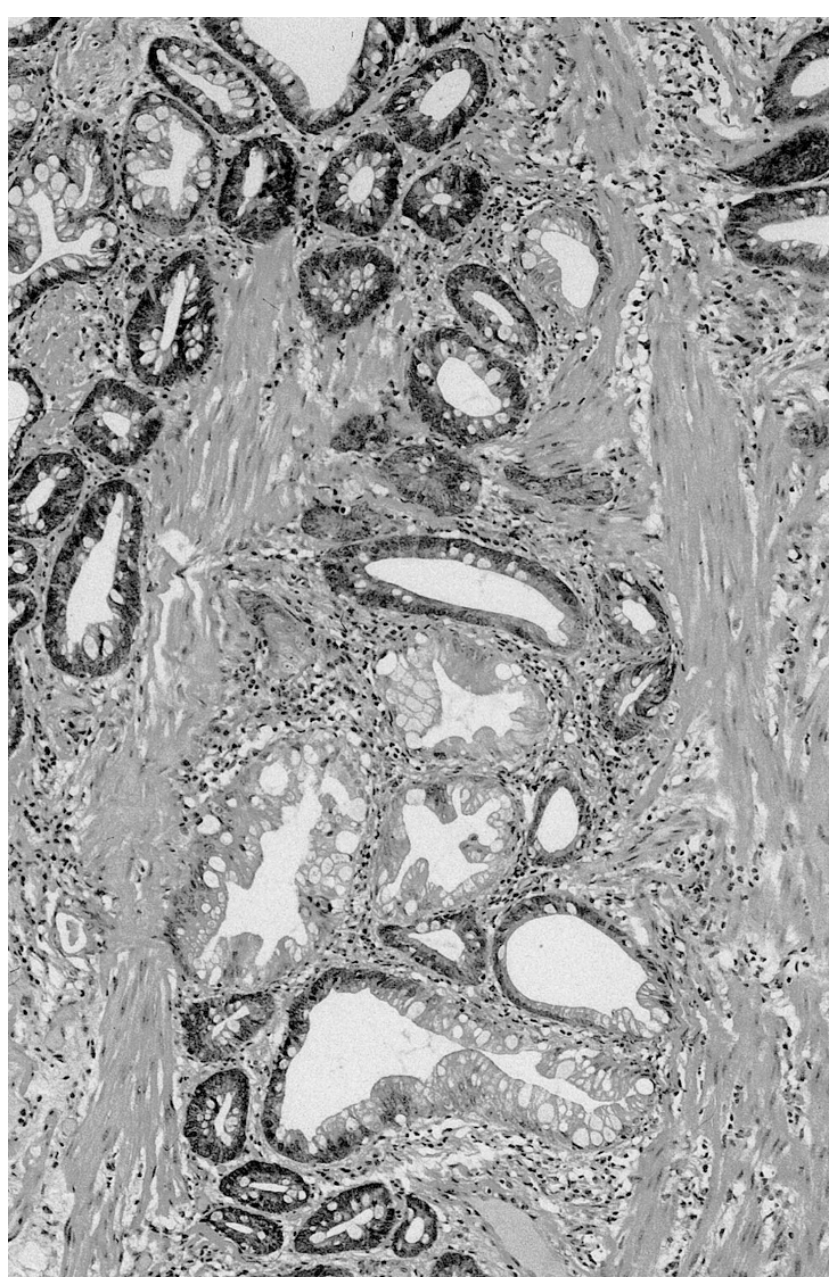

FIGURE 4. High-power view of a hyperplastic polyp with epithelial misplacement. Smooth muscle fibers of the muscularis mucosae surround lobules of misplaced epithelium with abundant lamina propria.

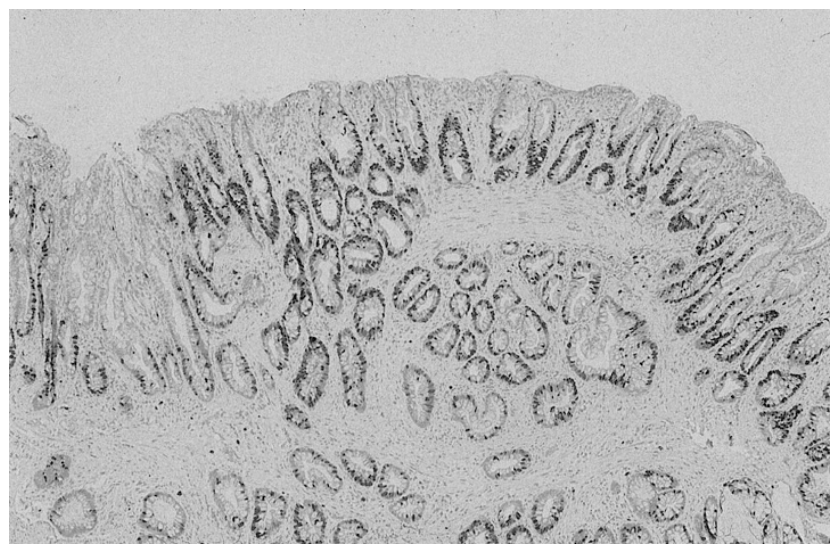

FIGURE 5. Immunohistochemical stain for MIB-1. Many of the misplaced crypts in the submucosa harbor cells that demonstrate strong nuclear staining for MIB-1 (bottom), similar to the basal zone of the mucosal portion of the polyp (top).

phologic and immunophenotypic properties of hyperplastic polyps with misplaced epithelium confirm the benign nature of the lesions and suggest that they may develop secondary to local tissue injury.

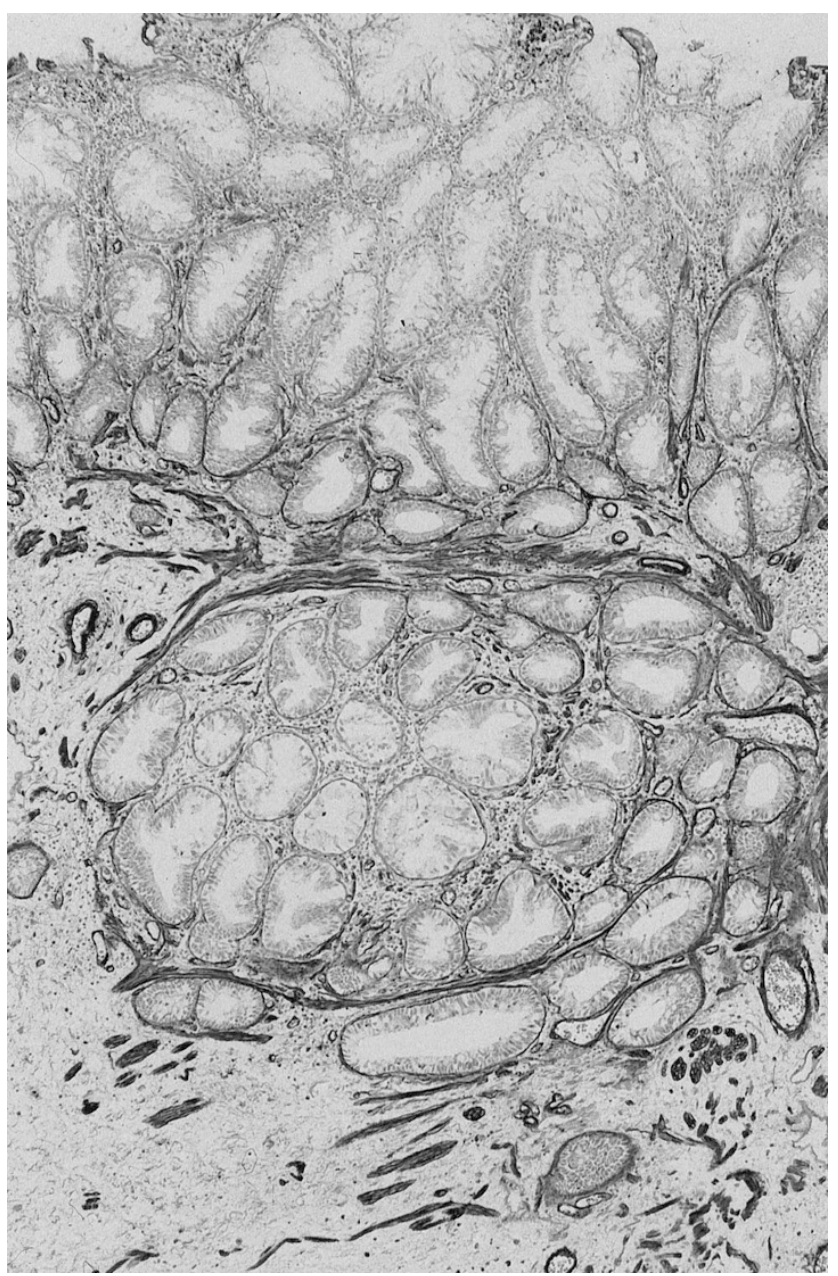

FIGURE 6. Immunohistochemical stain for collagen IV. Note the presence of a continuous pattern of basement membrane staining surrounding submucosal misplaced crypts (bottom). The pattern is similar to that seen in the mucosal part of the polyp (top).

Our study provides evidence to support the theory that these lesions result from local trauma. Morphologically, many of the polyps in this study showed recent hemorrhage, submucosal vascular congestion, and hemosiderin deposits, features that were not present in the adjacent nonpolypoid mucosa. Additionally, misplaced epithelium was always associated with areas of discontinuity in the muscularis mucosae. Furthermore, the pattern of MIB-1, E-cadherin, and collagen IV staining in misplaced epithelium was similar to that observed in the basally located crypts in the mucosal portion of the polyps, which also provides evidence in support of the above-noted theory of pathogenesis (13). Thus, on the basis of these findings, we believe that misplacement of crypt epithelium into the submucosa of hyperplastic polyps may occur via a mechanism similar to that observed in adenomas with so-called pseudoinvasion. In fact, we agree with Sobin $(12,14)$, who first suggested that misplacement of epithelium may occur secondary to tissue damage from torsion, or twisting, of the polyp followed by protrusion of glands through inherently 
weak regions of the muscularis mucosae, such as those that occur normally adjacent to lymphoid aggregates. Although none of the hyperplastic polyps in our study were pedunculated, it is possible that minor trauma may occur due to other factors, such as vigorous peristalsis or bulky intraluminal material.

A comprehensive literature search revealed only two other studies that evaluated lesions similar to those reported here. In 1985, Sobin (12) described 10 cases of "inverted" hyperplastic polyps morphologically similar to the lesions described in this study. However, in that study, the polyps were located predominantly in the right colon and were more common in females compared with conventional hyperplastic polyps. In contrast to the lesions reported by Sobin, the polyps in our study occurred in males and females equally and were located exclusively in the left colon. The reason for the difference in anatomic distribution between these two studies is unclear. Most of the patients in our study (92\%) had a full colonoscopy, with visualization of the cecum, which eliminates the possibility that the difference in anatomic distribution resulted from an incomplete evaluation of the colon.

In 1993, Shepherd (11) described two cases of multiple inverted hyperplastic polyps with histologic features similar to those observed in our study. However, in contrast to the patients reported herein, both patients in Shepherd's study were older males who had multiple $(>10)$ large $(0.4$ to $2.5 \mathrm{~cm})$ polyps located adjacent to a large, fungating right-colonic adenocarcinoma. Morphologically, the polyps in that report were slightly different in that they had a "pitted" surface and contained numerous large mucin-filled cysts reminiscent of colitis cystica profunda. Therefore, given the clinical and morphologic differences, it is unclear whether the polyps described in Shepherd's study represent a similar pathobiologic entity as the ones described here.

The presence of misplaced epithelium in hyperplastic polyps may lead to diagnostic confusion with a number of other entities, such as prolapse-type inflammatory polyps, adenomas with so-called pseudoinvasion, or even adenomas with invasive welldifferentiated adenocarcinoma. All of these lesions may contain epithelial elements within the submucosa of the polyp stalk. However, careful attention to histologic detail can readily differentiate these other entities from hyperplastic polyps with epithelial misplacement. For instance, prolapse-type inflammatory polyps are frequently inflamed or even ulcerated, contain markedly regenerative epithelium, and show characteristic fibromuscular hyperplasia of the lamina propria. In contrast, hyperplastic polyps with misplaced epithelium are largely composed of proliferating crypts without significant inflammation. At low magnification, an adenoma with pseudoinvasion may also look similar to a hyperplastic polyp with misplaced epithelium. However, adenomas are dysplastic lesions composed of neoplastic cells with pseudostratified, hyperchromatic, and enlarged nuclei, frequent mitoses, and abundant single cell necrosis. Furthermore, these lesions do not normally demonstrate epithelial maturation. Similarly, adenomas with invasive adenocarcinoma contain infiltrating crypts comprised of cells with markedly dysplastic nuclei, foci of necrosis, and abnormal mitotic figures, as well as a desmoplastic stroma.

In summary, we report the clinical, pathologic, and immunophenotypic features of an unusual morphologic variant of hyperplastic polyp that shows misplacement of crypt epithelium into the submucosa. We propose that these polyps may result from local trauma and subsequent protrusion of epithelium into the submucosa through inherently weak areas in the muscularis mucosae, such as that which occurs adjacent to lymphoid aggregates. We prefer the term $h y$ perplastic polyp with epithelial misplacement because this more clearly reflects our impression that the polyps develop through a similar pathogenetic mechanism as that proposed for adenomas with pseudoinvasion. Pathologists should be aware of this entity to avoid diagnostic confusion with other, more serious lesions.

\section{REFERENCES}

1. Araki K, Ogata T, Kobayashi M, Yatani R. A morphological study on the histogenesis of human colorectal hyperplastic polyps. Gastroenterology 1995;109:1468-74.

2. Arthur JF. The significance of small mucosal polyps of the rectum. Proc R Soc Med 1962;55:703-5.

3. Arthur JF. Structure and significance of metaplastic nodules in the rectal mucosa. J Clin Pathol 1968;21:735-43.

4. Morson BC. Precancerous lesions of the colon and rectum. JAMA 1962;179:316-21.

5. Morson BC. Some peculiarities in the histology of intestinal polyps. Dis Colon Rectum 1962;5:337-44.

6. Vatn MH, Stalsberg H. The prevalence of polyps of the large intestine in Oslo: an autopsy study. Cancer 1982;49:819-25.

7. Williams AR, Balasooriya BA, Day DW. Polyps and cancer of the large bowel: a necropsy study in Liverpool. Gut 1982;23:835-42.

8. Estrada RG, Spjut HJ. Hyperplastic polyps of the large bowel. Am J Surg Pathol 1980;4:127-33.

9. Goldman H, Ming S, Hickok DF. Nature and significance of hyperplastic polyps of the human colon. Arch Pathol 1970; 89:349-54.

10. Grondin MV, Chang WWL, Gaskins RD. Crypt alterations and collagen deposition in hyperplastic polyps of colorectum. Dig Dis Sci 1990;35:12-9.

11. Shepherd NA. Inverted hyperplastic polyposis of the colon. J Clin Pathol 1993;46:56-60.

12. Sobin LH. Inverted hyperplastic polyps of the colon. Am J Surg Pathol 1985;9:265-72.

13. Valizadeh A, Karayiannakis AJ, el-Hariry I, Kmiot W, Pignatelli M. Expression of E-cadherin associated molecules (alpha-, beta-, and gamma-catenins and p120) in colorectal polyps. Am J Pathol 1997;150:1977-84.

14. O'Leary AD, Sweeney EC. Lymphoglandular complexes of the colon: structure and distribution. Histopathology 1986; 10:267-83. 


\section{Book Review}

\section{Pollack GH: Cells, Gels, and the Engines of Life, 305 pp, Seattle, Ebner \& Sons, 2001, (\$27.95).}

Robert Hooke (1635-1703), the English physician, naturalist, and philosopher, is credited with establishing the cellular nature of living tissues in his "Micrographia" of 1665. Introductory botany courses (in my day at least) were prone to open with remarks on Hooke's depiction of a crosssection through a cork that he had popped out of a wine bottle. Although the good doctor was aware of significant differences between living and dead cells, it is an incongruity that he actually drew a magnified image of a mass of cell walls. The protoplasms and the plasma membranes of those cells had long disappeared, even at the time the bark was harvested from the living oak tree. Hooke had invented the balance spring for watches 7 years earlier, and he went on to write on a wide range of scientific subjects, including earthquakes. Given this combination, I dare say he would have been attracted to the engaging title of the present volume, written three centuries later by a bioengineer who is devoted to exploring nature's way of converting chemical energy into mechanical work at the cellular level.

All living protoplasm is bounded by a limiting membrane, which is composed of phospholipids, sterols and proteins, of various and variable compositions that reflect both the function and the milieu of the intact cell. Much of the biochemistry, biophysics, and ultrastructure of plasma membranes has been elucidated during the past century. However, Gerald Pollack maintains that there has been far too much scientific shouting about the pivotal role of these membranes in health and disease. He wants to place more emphasis on the cytoplasm and, in particular, the manifest properties of aqueous gels containing concentrated proteins, nucleic acids, and polysaccharides.

Accordingly, this book aims to convince the reader that much more attention should be paid to the biophysics of the cytoplasm and that many of the cell's impressive functions still can be performed under conditions where the membrane is stressed, leaky, or even broken. The arguments are built on the work of a handful of pioneers in this field, Gilbert Ling and Albert Szent-Györgyi and more recently Ichiji Tasaki and Gen Matsumoto, which is extended by Pollack into an intriguing scientific story. He starts as if walking on eggs (probably fearing that his audience is more peeved than titillated by his audacious hypothesis) and then carries us persuasively from chapter to chapter. Along the way Professor Pollack introduces much physics and chemistry in a "user-friendly" manner, and the wide outside margins are more often than not filled with successful illustrations, charts, and cartoons. The art work was ably executed by David Olsen.

The book is assembled in five sections. The first sets the stage by debunking myths and sharpening organized skepticism, and the second establishes the groundwork on water, solutes, ions, and cell potentials. The third and central section advances the hypothesis that cell function resembles gel function and emphasizes phase transitions as driving forces for diverse biological mechanisms. Here the author pulls out all the stops and presents much that is worthy of contemplation. The fourth part deals with cell dynamics and discusses everything from secretion to muscle contraction. The fifth section is entitled "loose ends" and attempts an integration of the underlying themes.

Toward the end there is a discussion on water transport in tall trees, capillary action, and the role of gels within the water conduction vessels (xylem). In fact, a little more attention to walled cells (algae, bacteria, and fungi as well as higher plants) would have complemented this book. Notwithstanding the power of the present arguments, anyone who has had the pleasure of watching under the light microscope the reversible plasmolysis of purple beetroot protoplasts (in and out of sucrose solution and distilled water) will still maintain a healthy respect for the function of plant plasma membranes and their mechanical protection by cell walls.

Gerald Pollack is Professor of Bioengineering at the University of Washington, Seattle. He presents a fresh view of how cells function, hopes to reach a wide audience, and displays an intense commitment to his subject. The phenomenon is important, the working hypothesis is contentious, but modern pathologists will enjoy the presentation and benefit from the exercise of reading this book.
Wilfred Niels Arnold
Biochemistry \& Molecular Biology
University of Kansas Medical Center
Kansas City, Kansas 